\title{
Site testing on the Greenland Ice Cap
}

\author{
M. I. Andersen ${ }^{1}$, K. Pedersen ${ }^{2}$ \& A. N. Sørensen ${ }^{2}$ \\ ${ }^{1}$ Dark Cosmology Centre, Niels Bohr Institute, Univ. Copenhagen, Juliane Mariesvej 30, \\ DK-2100 Kbh Ø, Denmark \\ email: mia@dark-cosmology.dk \\ ${ }^{2}$ Niels Bohr Institute, Univ. Copenhagen, Juliane Mariesvej 30, DK-2100 Kbh Ø, Denmark
}

\begin{abstract}
We present a site testing program initiated at the SUMMIT station on the Greenland Ice Cap. A DIMM was mounted in the SWISS tower, $39 \mathrm{~m}$ above the ice level, during a period of 3 weeks in the late Arctic summer 2008. Tracking Polaris, the DIMM obtained continuous seeing measurements. The campaign was hampered by poor weather and the measured seeing was fluctuating, suggesting that the boundary layer was very unstable. However, during short periods, the un-calibrated seeing went below 0.5 , indicating that the free atmosphere seeing above Greenland is not significantly different from what is found above the Antarctic plateau.
\end{abstract}

Keywords. Site Testing, Atmospheric Effects

\section{Introduction}

The Antarctic plateau has over the past 30 years been established as an observing platform of increasing importance (Indermuehle, Burton \& Maddison (2005)). The key parameters which makes sites on the Antarctic plateau, such as Dome C, Dome F and Dome A, interesting, is the excellent seeing above a very thin boundary layer (Lawrence et al. (2004), Aristidi et al. (2009)), the cold environment and the extremely low precipitable water vapor. A limitation of Antarctic is that only about one third of the sky is visible. A similar site in the Northern hemisphere could complement the Antarctic plateau.

\section{Greenland as an Astronomical Site}

The only approximately similar site in the Northern hemisphere is the Greenland Ice Cap. Its area is 7 times smaller than Antarctica, but the altitude of summit, the highest point on the ice cap, is 3,221 $\mathrm{m}$ and thus almost identical to Dome-C. Because the scale height of the boundary layer under stable high pressure conditions is many orders of magnitude smaller than the extent of these ice caps, there is no reason to believe that the behavior of the boundary layer above the Greenland Ice Cap is fundamentally different from what is observed above the Antarctic plateau. There is thus reason to believe that one can get access to the free atmosphere from a tower of manageable height.

As compared to the Antarctic plateau, the Greenland Ice Cap is much more affected by external weather systems. However, Greenland has 56,000 inhabitants and an infrastructure which works year around, with daily connections between Kangerlussuaq international airport and Copenhagen on commercial airlines. There is also access by sea all year. In the summer season there is access to SUMMIT via USAF C-130 planes. In the winter season there is access with Twin-Otter planes and medical evacuations can be carried out, except for a few days a year when the temperature at SUMMIT drops below $-55^{\circ} \mathrm{C}$. 


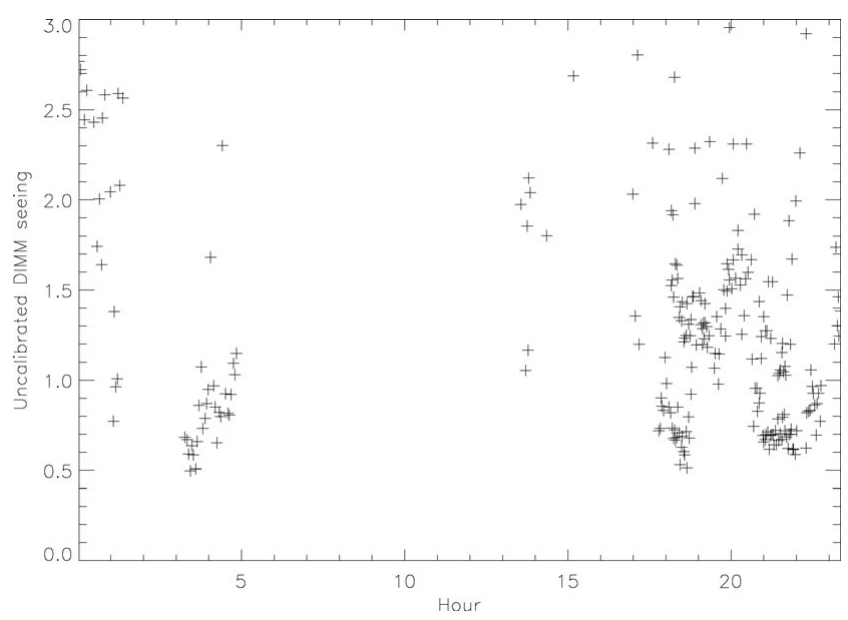

Figure 1. Uncalibrated seeing data for one day during the campaign. The measured differential image motion varies on short time scales, suggesting that the height of the boundary layer varied quite dramatically during these measurements.

\section{A Tower-Mounted Seeing Monitor}

The reference instrument for seeing measurement is the Differential Image Motion Monitor (DIMM) (Sarazin, M. \& Roddier, F. (1990)). The challenge in characterizing sites on ice plateaus with a DIMM is that it must be located above the boundary layer, which has a completely dominant contribution to the seeing. The strategy which was identified as likely to be most successful for measuring the free atmosphere seeing above the Greenland Ice Cap at SUMMIT was therefore to place a DIMM, which can track Polaris, in the $50 \mathrm{~m}$ high Swiss tower. This DIMM is pointed to the celestial pole and brings the light from Polaris onto the axis of the DIMM telescope through the use of achromatic prisms. The achromatic prisms are mounted in a wheel which rotates at the siderial rate. The DIMM is foreseen to be able to operate fully autonomously.

\section{First Seeing Measurements}

During three weeks in August 2008, in the late Arctic summer, the DIMM was deployed in the Swiss tower, $39 \mathrm{~m}$ above the ice. Unfortunately the weather was not co-operative. There were clouds during most of the days and more or less continuous data could only be taken during some part of one day. The DIMM has not yet been cross-calibrated against another DIMM, so only un-calibrated seeing measurements are shown in Fig. 1. After calibration, the best seeing measurements are likely $10 \%-20 \%$ better, i.e. closer to 0".4. Permanent installation of the DIMM is intended from 2010 or 2011.

\section{Acknowledgements}

We acknowledge support from the NSF Arctic programme at SUMMIT and from the Instrument Centre for Danish Astrophysics

\section{References}

Aristidi, E. et al. 2009 Astron. and Astrophys. 499, 955

Indermuehle, B., Burton, M., \& Maddison, S. 2005 PASA 22, 73

Lawrence, J. S. et al. 2004 NATURE 431, 278

Sarazin, M. \& Roddier, F. 1990 Astron. and Astrophys. 227, 294 